\title{
Polysaccharide-Based pH-Responsive Nanocapsules Prepared with Bio-Orthogonal Chemistry and Their Use as Responsive Delivery Systems
}

Mohammad Shafee Alkanawati, Richard da Costa Marques, Volker Mailänder, Katharina Landfester,* and Hélö̈se Thérien-Aubin*

Cite This: Biomacromolecules 2020, 21, 2764-2771

Read Online

ABSTRACT: Bio-orthogonal reactions have become an essential tool to prepare biomaterials; for example, in the synthesis of nanocarriers, bio-orthogonal chemistry allows circumventing common obstacles related to the encapsulation of delicate payloads or the occurrence of uncontrolled side reactions, which significantly limit the range of potential payloads to encapsulate. Here, we report a new approach to prepare $\mathrm{pH}$-responsive nanocarriers using dynamic bio-orthogonal chemistry. The reaction between a poly(hydrazide) crosslinker and functionalized polysaccharides was used to form a $\mathrm{pH}$-responsive hydrazone
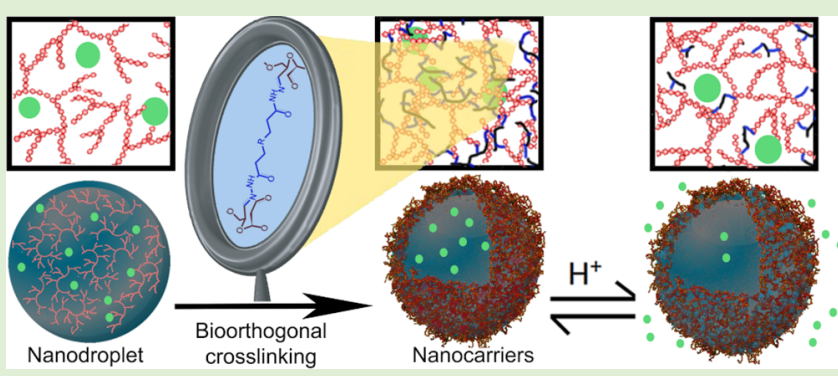
network. The network formation occurred at the interface of aqueous nanodroplets in miniemulsion and led to the production of nanocapsules that were able to encapsulate payloads of different molecular weights. The resulting nanocapsules displayed low cytotoxicity and were able to release the encapsulated payload, in a controlled manner, under mildly acidic conditions.

\section{INTRODUCTION}

The lack of selectivity during the preparation of biomaterials is a significant obstacle in the development of new functional materials for biomedical applications such as nanocarriers (NCs). Bio-orthogonal chemistries, reactions occurring uniquely, or at least preferentially, between functional groups of synthetic origin that can proceed under physiological conditions without interfering with the activity of other functional groups of the biomolecules, are an attractive solution to this lack of selectivity. ${ }^{1}$ Those reactions have become an essential tool for the labeling, conjugation, and surface modification of biomolecules because of their chemical selectivity and biocompatibility. ${ }^{2-4}$ Implementing such chemistries in the design of new polymer NCs can potentially allow for the encapsulation of delicate payloads. ${ }^{5-7}$

Polymer NCs prepared by polycondensation at the interface of a water-in-oil miniemulsion are promising candidates for gene and drug delivery. ${ }^{8,9}$ The advantages of these NCs in comparison to other nanovehicles are their relatively low polymer content for the formation of a capsule shell and high loading capacity. ${ }^{10-12}$ The interfacial crosslinking reaction of inverse-miniemulsion droplets is an ideal technique to encapsulate a variety of hydrophilic payloads. ${ }^{12-14}$ However, in the past, their preparation usually relied on nonselective reactions, which are detrimental to the final behavior of the NCs such as the efficacy of the release of the payload or to the biodegradability of the carriers. For example, the reaction between multifunctional alcohols, amines, or thiols and diisocyanate compounds has shown the efficient encapsulation of a variety of payloads in polyurea, polyurethane, or polythiourea nanocapsules. ${ }^{15,16}$ However, such reactions involving nucleophiles like alcohols and amines that are also present in sensitive biomolecules used as payloads could lead to side reactions and cause a partial or total decrease in the efficacy of the payload.

A method to avoid unwanted side-reactions consists in integrating bio-orthogonal chemistry to the interfacial synthesis of NCs, and this approach would be beneficial to the development of new and more efficient NCs for drug release or gene therapy. ${ }^{17}$ Bio-orthogonal reactions such as olefin metathesis and copper-catalyzed azide-alkyne click reaction have been successfully used to prepare nanocarriers. ${ }^{18-20}$ Although these chemistries displayed the desired selectivity, they still required the use of catalysts; this led to the implementation of catalyst-free reactions in the development of nanocapsules; for example, the copper-free click-reaction between a tetrazole functionalized protein and crosslinker functionalized with cyclic alkenes was successfully used to

Received: April 3, 2020

Revised: June 9, 2020

Published: June 12, 2020 


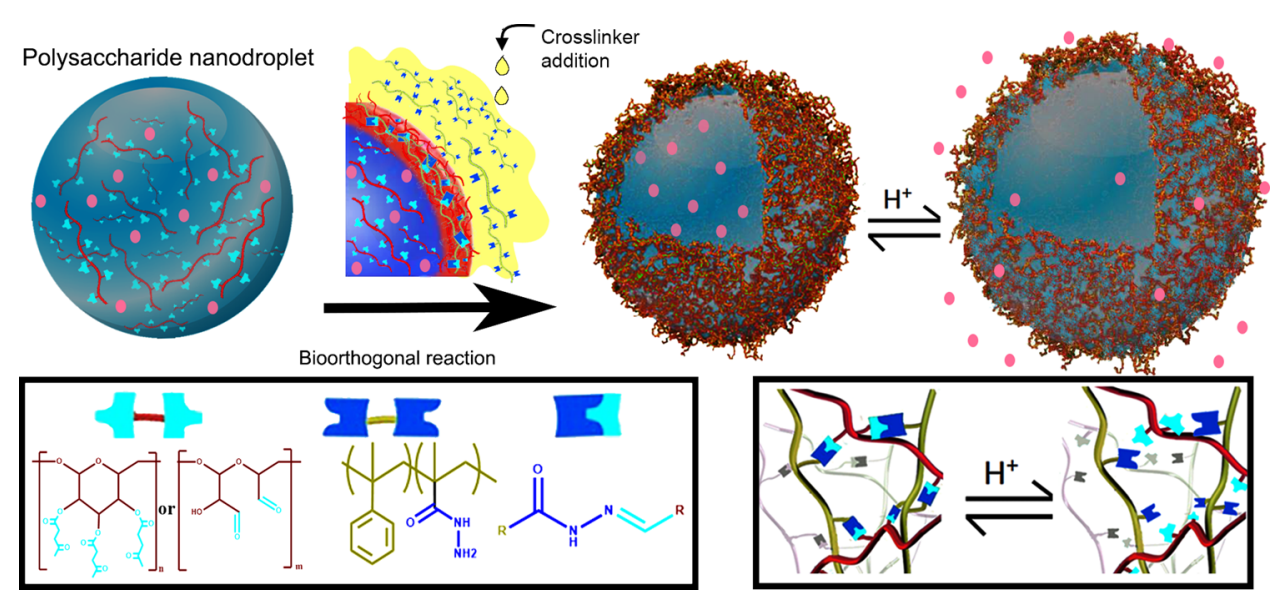

Figure 1. Synthesis of dextran-based $\mathrm{pH}$-responsive and bio-orthogonal hydrazone nanocarriers.

prepare NCs. ${ }^{7}$ These reactions are attractive strategies for the successful encapsulation of biomolecules and therapeutic substances. However, milder alternatives and more compliant bio-orthogonal chemistries still need to be implemented in the production of NCs to offer the broad range of reaction conditions needed to satisfy the requirements for the encapsulation of different complexes and sensitive payloads. One of such chemistries would be to use the reaction between hydrazide and ketone to form a crosslinked NC shell. This reaction occurs in aqueous media, at a physiological $\mathrm{pH}$ value and room temperature without the addition of any chemical, leads to the formation of a hydrazone ligation, and has been used for the labeling of protein, ${ }^{21}$ DNA, RNA, ${ }^{22}$ and cells. ${ }^{23}$ Furthermore, interestingly for the design of drug delivery devices, the hydrazone bond has a limited hydrolytic stability and can dissociate under acidic conditions. ${ }^{24-26}$

Here, to prepare NCs compliant with the delivery of active molecules within weak acidic environments such as tumor sites, inflammatory tissues, or intracellular compartments like endosomes and lysosomes, the interfacial reaction between a poly(aldehyde) or poly(ketone), derived from polysaccharides, dissolved in water nanodroplets and an oil-soluble poly(hydrazide) was used to create the shell of responsive NCs by interfacial polycondensation (Figure 1). Furthermore, since the $\mathrm{pH}$ value of the environment can influence the hydrazone network created, it was used, as a result, for the controlled release of the payload. ${ }^{27}$ The bio-orthogonal reaction between reactive carbonyl and acyl hydrazine led to the synthesis of $\mathrm{pH}$ responsive NCs based on functionalized dextran. Additionally, the release from the resulting NCs was controlled, under physiologically relevant conditions, by the degree of crosslinking of the hydrazone network tuned by the $\mathrm{pH}$-responsive nature of equilibrium between hydrazide and hydrazone.

\section{EXPERIMENTAL SECTION}

Synthesis of the Nanocarriers. The precursors for the synthesis of the NCs, aldehyde-functionalized dextran $(\mathrm{OxD})$, ketone-functionalized dextran $(\mathrm{KeD})$, and poly(styrene-co-methacryloyl hydrazide) (PSH), were prepared as described in the Supporting Information (Figures $\mathrm{S} 1-\mathrm{S} 3$ and Table S1). The NC synthesis was carried out by the interfacial reaction between functionalized dextran, either $\mathrm{OxD}$ or $\mathrm{KeD}$, in aqueous droplets, and PSH in the continuous toluene phase. The water phase was prepared by dissolving $150 \mathrm{mg}$ of functionalized dextran and $16 \mathrm{mg}$ of $\mathrm{NaCl}$ in $1 \mathrm{~g}$ of phosphate buffer solution (20 $\mathrm{mM})$. For the NCs used for in vitro studies, a mixture of $5 \mathrm{wt} \%$ of Cy5-labeled $\mathrm{OxD}($ or $\mathrm{KeD}$ ) and 95 wt \% of unlabeled $\mathrm{OxD}$ (or $\mathrm{KeD}$ ) was used. As a model payload, $5 \mathrm{mg}$ of rhodamine-labeled dextran of different molecular weights (either 150 or $500 \mathrm{kDa}$ ) was added to the aqueous phase. The continuous oil phase was prepared by adding 160 $\mathrm{mg}$ of PGPR to $10 \mathrm{~g}$ of toluene. The oil phase was added dropwise to the stirred water phase to form an inverse pre-emulsion. Then, the pre-emulsion was passed 2 times through a microfluidizer (LV1) equipped with a Y-shape interaction chamber with $75 \mu \mathrm{m}$ channels at a pressure of $896.3 \mathrm{bar}$, resulting in the formation of a miniemulsion. A second oil phase was prepared by dissolving $90 \mathrm{mg}$ of PGPR and $150 \mathrm{mg}$ of $\mathrm{PSH}$ in $4 \mathrm{~g}$ of toluene. After the dropwise addition of the second oil phase to the stirred miniemulsion, the reaction mixture was stirred for $24 \mathrm{~h}$ at room temperature. The NCs were purified by two cycles of centrifugation (1770 RCF) followed by redispersion in pure toluene. To transfer the NCs to water, $1 \mathrm{~g}$ of the toluene dispersion was added dropwise, under gentle sonication, to $3 \mathrm{~g}$ of a $0.1 \mathrm{wt} \%$ solution of sodium dodecylsulfate (SDS) in PBS buffer. After the addition, the samples were stirred in open vials for $6 \mathrm{~h}$ at room temperature to evaporate the toluene. Then, the excess SDS was removed using centrifugal concentrators (pore size $1000 \mathrm{kDa}$ ) followed by redispersion in fresh PBS buffer.

Release Kinetics. The release of the payload was measured by fluorescence spectroscopy after the centrifugal ultrafiltration of the nanocapsule suspensions incubated for different periods of time in buffer solution at controlled $\mathrm{pH}$ values. After the transfer of the NCs to water, $10 \mathrm{~mL}$ of the suspension was filtered by centrifugal ultrafiltration at $1770 \mathrm{RCF}$ using Vivaspin $1000 \mathrm{~K}$ centrifugal concentrators. The NCs collected on the filter were redispersed in $4 \mathrm{~mL}$ of phosphate buffer $\mathrm{pH}=7.4$, yielding a suspension of NCs of ca. $3 \mathrm{mg} / \mathrm{mL}$. Then, after appropriate time intervals $(0,0.5,1$, and 3 h), $250 \mu \mathrm{L}$ of the suspension was taken out and filtered by centrifugal ultrafiltration at $1770 \mathrm{RCF}$ for 15 min using a spin filter (vivaspin 500 $\mu \mathrm{L} 1000 \mathrm{~K}$ ), and the fluorescence of the filtrate was measured at $\lambda_{\mathrm{ex}}=$ $548 \mathrm{~nm}$ and $\lambda_{\mathrm{em}}=576 \mathrm{~nm}$.

After $3 \mathrm{~h}$, the sample was split in three aliquots of $1 \mathrm{~mL}$, and each aliquot was diluted by the addition of $2 \mathrm{~mL}$ of a solution at the appropriate $\mathrm{pH}$ value to yield final suspensions of a concentration of ca. $1 \mathrm{mg} / \mathrm{mL}$ at a $\mathrm{pH}$ value of $7.4,6$, or 5.2. Then, at appropriate time intervals $(0,1,3,6,24,48,72,96,112,136$, and $168 \mathrm{~h}), 250 \mu \mathrm{L}$ of the suspension was taken out and centrifuged at $1770 \mathrm{RCF}$ for $15 \mathrm{~min}$ using a spin filter (vivaspin $500 \mu \mathrm{L} 1000 \mathrm{~K}$ ). The concentration of the payload released was measured from the fluorescence intensity of the filtrate.

Cell Viability and Cellular Nanocarrier Uptake. The viability of the cells was quantified with the CellTiter-Glo luminescent cell viability assay (Promega, Germany) after coincubation of the cells with the NC suspension with a concentration ranging from 37.5 to $300 \mu \mathrm{g} / \mathrm{mL}$ for 2,24 , and $48 \mathrm{~h}$. The uptake of the NCs by the HeLa cells was quantified by flow cytometry after the coincubation of the cells with a suspension of $75 \mu \mathrm{g} / \mathrm{mL}$ for 2,24 , and $48 \mathrm{~h}$. The uptake was also visualized by confocal fluorescence microscopy after $48 \mathrm{~h}$ of 
the coincubation of the cells with a suspension of NCs at a concentration of $75 \mu \mathrm{g} / \mathrm{mL}$. Details of the in vitro experiments are described in the Supporting Information.

\section{RESULTS AND DISCUSSION}

The NCs were prepared by the crosslinking of functionalized dextran, either aldehyde-functionalized dextran $(\mathrm{OxD})$ or

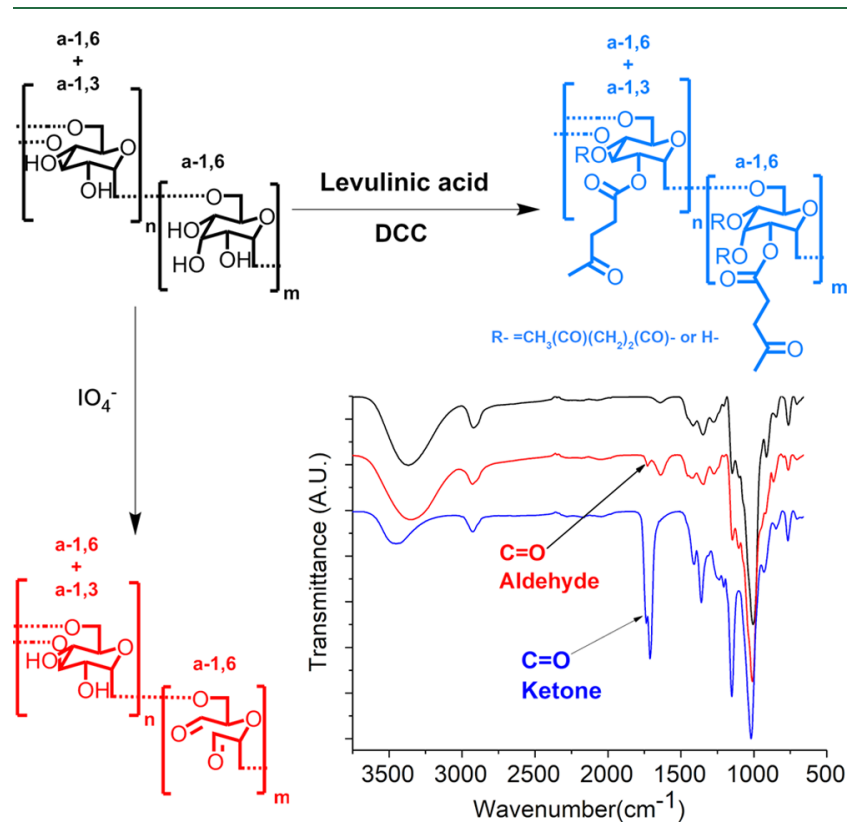

Figure 2. Dextran functionalized either with aldehyde groups $(\mathrm{OxD})$ (red) or with ketone groups (KeD) (blue).

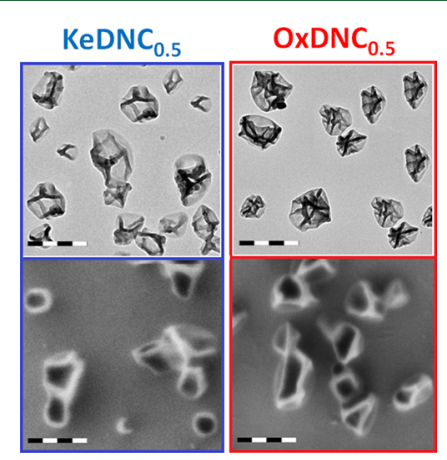

Figure 3. (A, B) TEM and (C, D) SEM images of the $\mathrm{KeDNC}_{0.5}$ and $\mathrm{OxDNC}_{0.5}$ nanocarriers. The scale bars are $200 \mathrm{~nm}$. ketone-functionalized dextran (KeD) (Figure 2) with poly(styrene-co-methacryloyl hydrazide) (PSH). The reaction of the functionalized dextran with the PSH resulted in the formation of a network of hydrazone linkages. To prepare the NCs, the dextran derivatives were dissolved in water and the $\mathrm{PSH}$ was dissolved in toluene. Then, the crosslinking reaction between the dextran derivatives and the PSH occurred at the interface of the stable aqueous nanodroplets in miniemulsion. This resulted in the formation of nanocapsules of uniform size and limited size distribution (Figure 3, Figure S4). Table 1 summarizes the properties of the resulting nanocapsules. The size of the NCs was controlled by the size of the parent precursor droplets (Figure S5), itself tunable by controlling the emulsification conditions. ${ }^{16,28}$ The nanocapsules prepared with the aldehyde-functionalized dextran $(\mathrm{OxD})$ were labeled $\mathrm{OxDNC}$, the nanocapsules prepared with the ketone-functionalized dextran $(\mathrm{KeD})$ were labeled $\mathrm{KeDNC}$, and nanocapsules prepared with a mixture of $\mathrm{OxD}$ and $\mathrm{KeD}$ were labeled $\mathrm{OxD}_{x} \mathrm{KeD}_{y} \mathrm{NC}$, where " $x$ " and " $y$ " are the weight fractions of $\mathrm{OxD}$ and $\mathrm{KeD}$, respectively. Furthermore, nanocarriers were prepared by the addition of different molar ratios of hydrazide and reactive carbonyls; during the preparation of $\mathrm{OxDNC}_{z}$ " $z$ " molar equivalents of hydrazide groups were added to the continuous phase in comparison to the water phase containing 1 molar equivalent of aldehydes.

Independent of the dextran derivative used, the size of the NCs in toluene was ca. $200 \mathrm{~nm}$ (Table 1). The size of the NCs was not significantly affected by the ratio of crosslinker molecules in the feed; similarly, the dry thickness of the shell of the NCs remained constant (Figure S6). After transfer to water, the NCs systematically swelled. As the molar ratio of the crosslinker to reactive groups on the dextran increased, a moderate decrease in the swelling was observed (Table 1), likely caused by the increase in the hydrophobic nature of the crosslinked network induced by the incorporation of more polystyrene-based crosslinkers and the increase in the crosslinking density of the shell. After transfer to water, the NCs also displayed a negative zeta potential attributed to remaining traces of the surfactant (SDS) used during the transfer of the NCs to water. Furthermore, those NCs showed excellent stability in aqueous media, and no significant changes in size and size distribution were observed even 6 months after their transfer to PBS buffer (Figure S7).

The preparation of the NCs was adjusted to yield the highest apparent encapsulation efficiency of model payloads. The apparent encapsulation efficiency was measured by the fraction of the payload used in the preparation of the dispersed

Table 1. Characteristics of the Crosslinked Dextran Nanocarriers Synthesized

\begin{tabular}{|c|c|c|c|c|c|c|c|}
\hline \multirow[b]{2}{*}{ nanocarrier } & \multirow[b]{2}{*}{ dextran } & \multirow[b]{2}{*}{ hydrazide /reactive carbonyl ratio } & \multicolumn{2}{|c|}{ average size $(\mathrm{nm})$} & \multicolumn{2}{|c|}{ PDI } & \multirow[b]{2}{*}{ zeta potential $(\mathrm{mV})$} \\
\hline & & & toluene & water & toluene & water & \\
\hline $\mathrm{OxDNC}_{0.25}$ & OxD 100 wt \% & 0.25 & 190 & 270 & 0.16 & 0.29 & -8.8 \\
\hline $\mathrm{OxDNC}_{0.5}$ & & 0.5 & 230 & 294 & 0.19 & 0.20 & -7.25 \\
\hline $\mathrm{OxDNC}_{1}$ & & 1 & 200 & 260 & 0.15 & 0.13 & -3.66 \\
\hline $\mathrm{OxDNC}_{1.5}$ & & 1.5 & 211 & 250 & 0.17 & 0.20 & -1.55 \\
\hline $\mathrm{KeDNC}_{0.25}$ & KeD 100 wt \% & 0.25 & 200 & 320 & 0.21 & 0.20 & -18.4 \\
\hline $\mathrm{KeDNC}_{0.5}$ & & 0.5 & 208 & 250 & 0.20 & 0.15 & -15.2 \\
\hline $\mathrm{KeDNC}_{1}$ & & 1 & 210 & 290 & 0.20 & 0.17 & -11.3 \\
\hline $\mathrm{KeDNC}_{1.5}$ & & 1.5 & 226 & 260 & 0.22 & 0.23 & -1.14 \\
\hline $\mathrm{OxD}_{50} \mathrm{KeD}_{50} \mathrm{NC}_{0.5}$ & $\mathrm{OxD} 50$ wt $\%+\mathrm{KeD} 50$ wt $\%$ & 0.5 & 190 & 250 & 0.19 & 0.25 & -21.4 \\
\hline $\mathrm{OxD}_{25} \mathrm{KeD}_{75} \mathrm{NC}_{0.5}$ & OxD 25 wt $\%+\mathrm{KeD} 75$ wt $\%$ & 0.5 & 201 & 280 & 0.21 & 0.26 & -20.0 \\
\hline
\end{tabular}



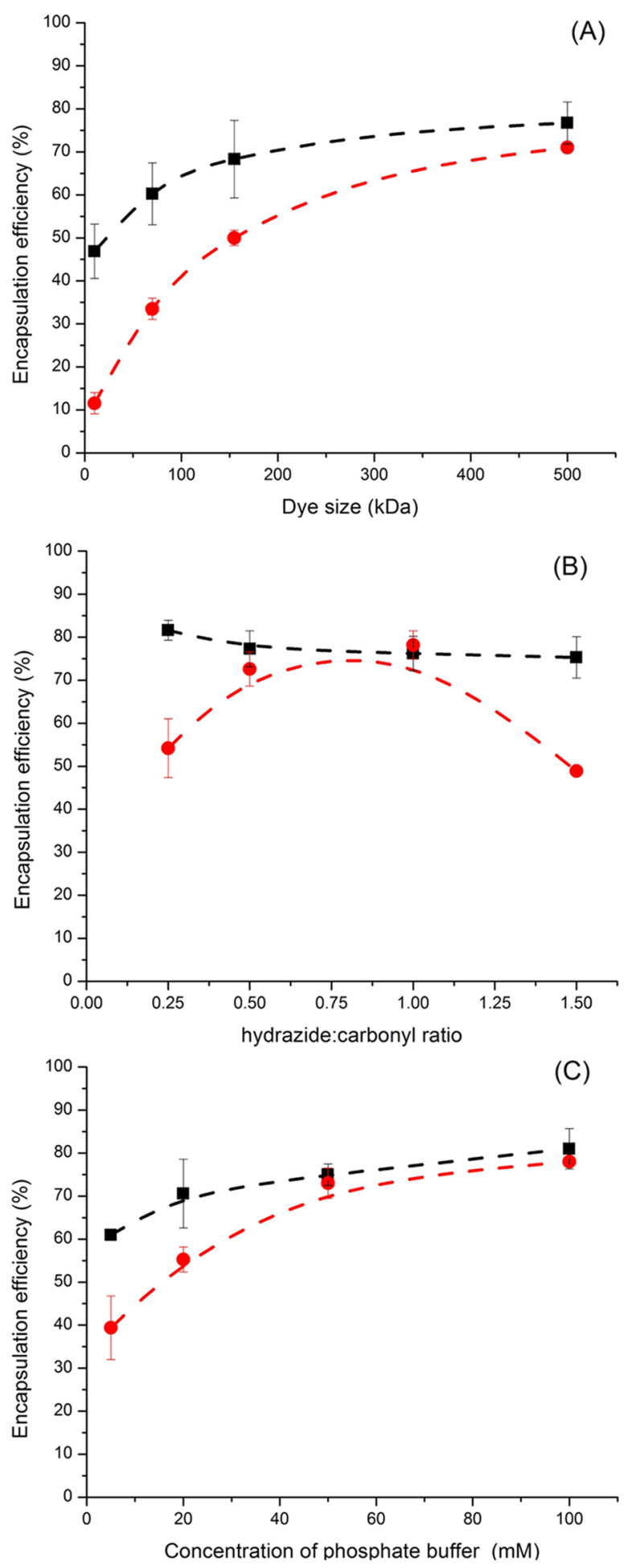

Figure 4. Encapsulation efficiency of the payloads in the nanocarriers prepared under different conditions. (A) Influence of the size of the model payloads, a series of dextran of different molecular weights functionalized with rhodamine, on their encapsulation in $\mathrm{OxDNC}_{0.5}$ (black) and $\mathrm{KeDNC}_{0.5}$ (red). (B) Influence of the stoichiometry of hydrazide and reactive carbonyl used during the synthesis of the nanocarriers for $\mathrm{OxDNC}_{x}$ (black) and $\mathrm{KeDNC}_{x}$ (red) on the encapsulation of rhodamine-functionalized dextran $\left(M_{n}: 500 \mathrm{kDa}\right)$. (C) Influence of the concentration of the phosphate buffer used in the precursor nanodroplets for $\mathrm{OxDNC}_{0.5}$ (black) and $\mathrm{KeDNC}_{0.5}$ (red) on the encapsulation of rhodamine-functionalized dextran $\left(M_{n}: 500\right.$ $\mathrm{kDa}$ ). phase present in the NCs after their transfer and equilibration in PBS buffer and accounts for both the payload molecules that were not encapsulated and those released in the first $6 \mathrm{~h}$ following the transfer to water (corresponding to the time needed to complete the water transfer process). First, a series of dextrans of different molecular weights functionalized with rhodamine were used to determine the molecular weight cutoff for the successful encapsulation in the $\mathrm{OxDNC}_{0.5}$ and $\mathrm{KeDNC}_{0.5}$ (Figure 4A). Only macromolecular payloads could efficiently be loaded in the nanocapsules, in addition to the model dextran with different molecular weights; other molecules such as albumin $\left(\mathrm{M}_{\mathrm{n}}: 67 \mathrm{kDa}\right)$ were also encapsulated in both $\mathrm{OxDNC}$ and KeDNC (Figure S8). The apparent encapsulation of payloads was systematically more efficient in the aldehyde-functionalized $\mathrm{OxDNC}_{0.5} \mathrm{~s}$ than in the ketone-functionalized $\mathrm{KeDNC}_{0.5} \mathrm{~s}$, likely because the equilibrium constant for the reaction of an acyl hydrazide with an aldehyde is typically larger than that for the reaction with a ketone; $^{29}$ thus, the reaction of the polyaldehyde precursor with the polyhydrazide crosslinker formed a denser and more heavily crosslinked network in comparison to the reaction of the polyketone in the same conditions. ${ }^{30}$ This led to the more efficient encapsulation of smaller payloads in the OxDNCs than in the KeDNCs.

Because of the existence of this dynamic equilibrium between reacted and unreacted hydrazine and reactive carbonyl moieties, the stoichiometry of the reactant present during the reaction also affects the formation of a dense crosslinked network. $^{24}$ The results show (Figure 4B) that having an equimolar amount of ketone and hydrazide led to the highest encapsulation efficiency of the payloads. Furthermore, the ionic strength of the environment also influenced the encapsulation efficiency (Figure 4C); an increase in the concentration of the phosphate buffer used during the preparation of the $\mathrm{OxDNC}_{0.5}$ and $\mathrm{KeDNC}_{0.5}$ resulted in an improved encapsulation efficiency of the payloads, likely because of the accelerated reaction kinetic of hydrazone formation in the presence of ions. ${ }^{31,32}$

The acylhydrazone crosslinking points formed by the reaction of the functionalized dextran and the $\mathrm{PSH}$ are dynamic covalent bonds, which can undergo a reversible disassembly when the environment is changed. ${ }^{33}$ The assembly and disassembly of the acylhydrazone bonds in the network can be controlled by changing the $\mathrm{pH}$ value of the environment and can be used to trigger the release of the cargo. The release of the payload from the NCs was the result of the diffusion of the payload through the semi-permeable shell of the NCs. The permeability of the membrane was mostly affected by the crosslinking density, which can be tuned by the amount of crosslinking agent used but also by the $\mathrm{pH}$ value of the environment because of the dynamic nature of the hydrazine network. To study the stability of the NCs at different $\mathrm{pH}$ values and to study the effect of the acidity on the stability of the acylhydrazone network, NCs were prepared with either aldehyde-functionalized dextran $\left(\mathrm{OxDNC}_{0.5}\right)$ or with ketone-functionalized dextran $\left(\mathrm{KeDNC}_{0.5}\right)$, and the resulting NCs were transferred to PBS buffer $\mathrm{pH}=7.4$ at a concentration of ca. $3 \mathrm{mg} / \mathrm{mL}$. The release from those NCs was studied over $3 \mathrm{~h}$, and for all NCs, no or very limited release of the encapsulated payload was measured. Then, the suspension of NCs was diluted by the addition of 2-fold of acidic buffer, and the final $\mathrm{pH}$ value of the suspension was 
(A.1)
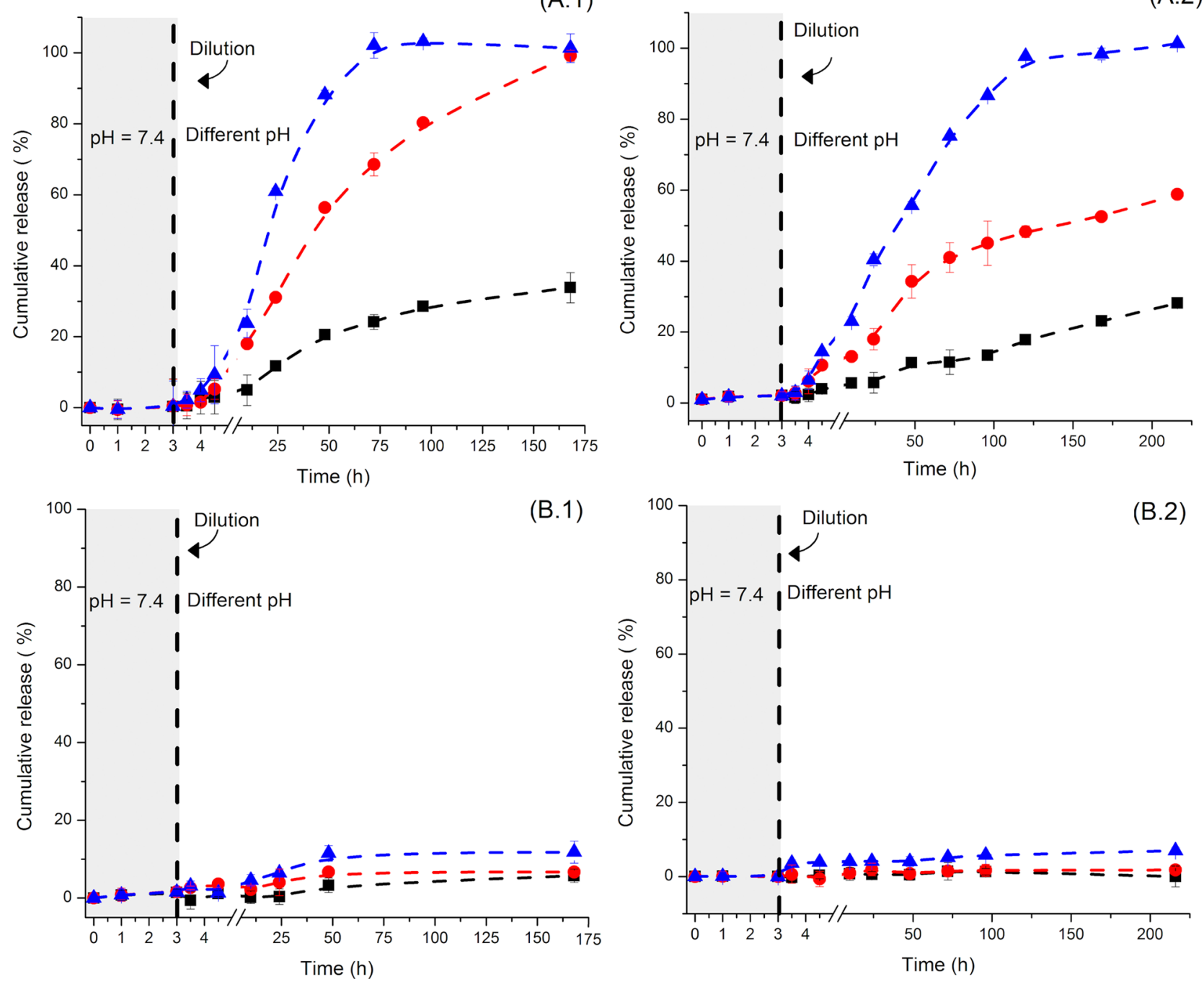

Figure 5. Effect of the $\mathrm{pH}$ value of the environment on the release of payloads from NCs prepared with (A) ketone-functionalized dextran $\left(\mathrm{KeDNC}_{0.5}\right)$ and $(\mathrm{B})$ aldehyde-functionalized dextran $\left(\mathrm{OxDNC}_{0.5}\right)$. Using rhodamine-functionalized dextran as the payload with a molecular weight (1) $\mathrm{M}_{\mathrm{n}}=150 \mathrm{kDa}$ and $(2) \mathrm{M}_{\mathrm{n}}=500 \mathrm{kDa}$. Measured at a $\mathrm{pH}$ value of $7.4(\boldsymbol{\square}), 6.0(\boldsymbol{\bullet})$, and $5.2(\boldsymbol{\Delta})$.

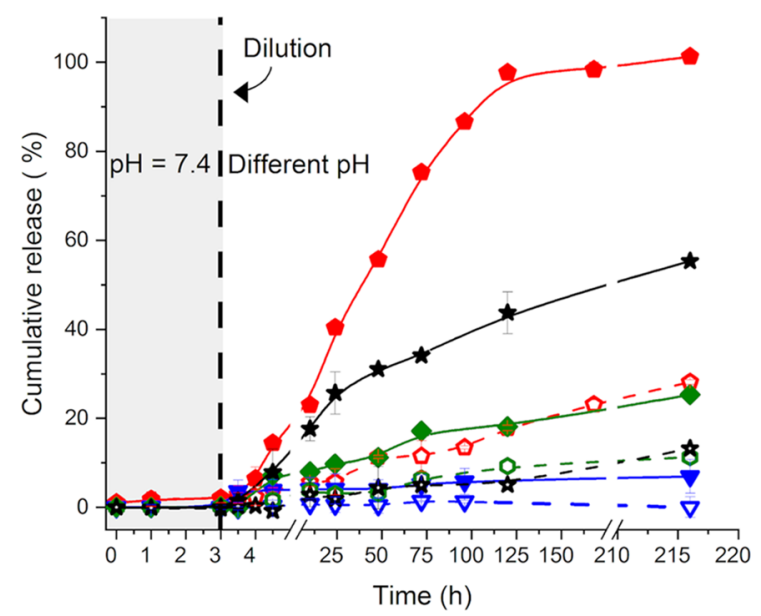

Figure 6. Effect of the composition of the dextran nanocarriers on the release of the encapsulated payload $\left(\mathrm{M}_{\mathrm{n}}=500 \mathrm{kDa}\right)$. For $\mathrm{OxD}_{50} \mathrm{KeD}_{50} \mathrm{NC}_{0.5}(\bullet), \mathrm{OxD}_{25} \mathrm{KeD}_{75} \mathrm{NC}_{0.5}(\star), \mathrm{KeDN}_{0.5}(\boldsymbol{\bullet})$, and $\mathrm{OxDN}_{0.5}(\boldsymbol{\nabla})$ at $\mathrm{pH}$ values of 7.4 (open symbols, dashed lines) and 5.2 (filled symbols, solid lines). either $5.2,6$, or 7.4 at a concentration of ca. $1 \mathrm{mg} / \mathrm{mL}$ of $\mathrm{NCs}$ in the suspension.

The type of reactive carbonyl group used, ketone or aldehyde, influenced the release kinetic as much as the $\mathrm{pH}$ value of the environment. NCs prepared with the $\mathrm{OxD}$ displayed a minimal release of the cargos at every $\mathrm{pH}$ value studied (Figure 5B). Inversely, the NCs prepared with $\mathrm{KeD}$, displayed a release of 5 to $10 \%$ of the cargo after $24 \mathrm{~h}$ in suspensions at a $\mathrm{pH}$ value of 7.4 , and between 45 and $60 \%$ of the cargo released when in suspensions at a $\mathrm{pH}$ value of 5.2. The increased release of the payload observed under acidic conditions was ascribed to the disassembly of the acylhydrazone crosslinking points and an effective decrease in the crosslinking density of the network in acidic media leading to an increased permeability of the shell of the NCs.

The cumulative release of the cargo from $\mathrm{KeDNC}_{0.5}$ was slow at neutral $\mathrm{pH}$ but increased significantly as the $\mathrm{pH}$ value of the suspension decreased (Figure 5A). At a pH value of 5.2, the cumulative release increased faster than that at a $\mathrm{pH}$ value of 6. This phenomenon occurred due to an increased dissociation of the acid-sensitive acylhydrazone bonds when the $\mathrm{pH}$ value decreased. The release from $\mathrm{OxDNC}_{0.5}$ (Figure 


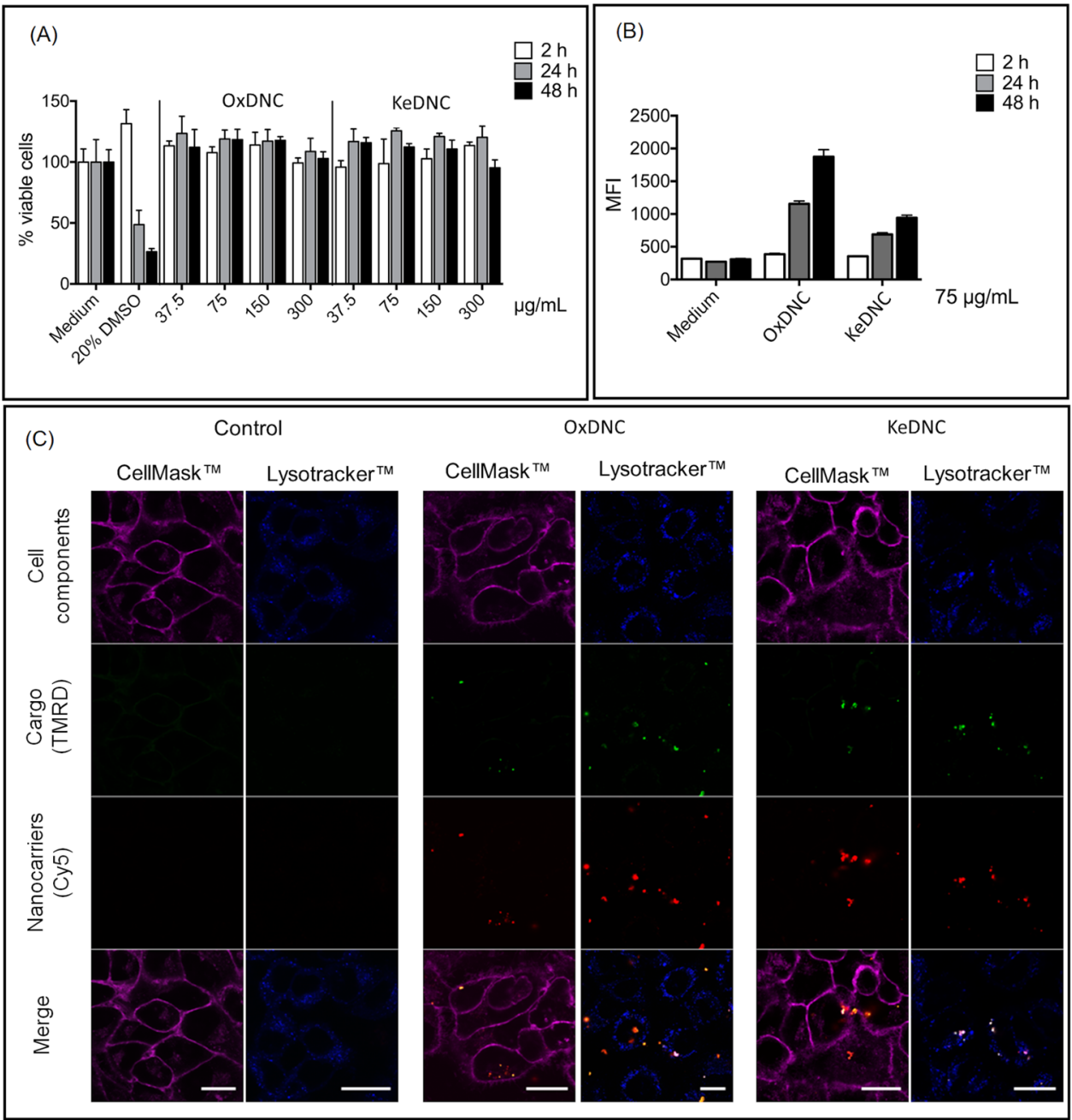

Figure 7. (A) Cell viabilities of HeLa cells after incubation with $\mathrm{KeDNC}_{0.5}$ and $\mathrm{OxDNC}_{0.5}$ measured after the incubation in cell media containing 37.5 to $300 \mu \mathrm{g} / \mathrm{mL}$ of nanocarriers. (B) Cellular uptake of $\mathrm{KeDNC}_{0.5}$ and $\mathrm{OxDNC}_{0.5}$ in HeLa cells after incubation for 2, 24, and $48 \mathrm{~h}$ in media containing $75 \mu \mathrm{g} / \mathrm{mL}$ of nanocarriers. (C) Confocal fluorescence images of HeLa cells after $48 \mathrm{~h}$ of incubation with media containing $75 \mu \mathrm{g} / \mathrm{mL}$ of nanocarriers. The scale bars are $20 \mu \mathrm{m}$.

5B) was only slightly responsive to changes in the acidity of the suspension. These results can be attributed to the higher thermodynamic stability of the acylhydrazone bonds formed between aldehyde and hydrazide in comparison to those formed between ketone and hydrazide. ${ }^{30,34}$ Figure 5 also highlights the effect of size on the release of the payload from the NCs. When the NCs were in an equilibrated state, for the first $3 \mathrm{~h}$ of the release experiment, both the rhodaminefunctionalized dextran with a molecular of $150 \mathrm{kDa}$ and the rhodamine-functionalized dextran with a molecular of $500 \mathrm{kDa}$ were efficiently entrapped inside the NCs. Once the system was diluted and acidified, both diffusion probes were released. Still, the release rate of the smaller probe was significantly higher (Figure S9) because the crosslinked network obstructed more efficiently the mass transport of the largest probes. ${ }^{35}$ While the presence of the hydrazone network enables the release triggered by an increase in the acidity of the NC environment, the release also occurred in the presence of dextranase, an enzyme able to degrade the functionalizeddextran backbone and destroy the NCs (Figure S8).

The cumulative release of payloads from the NCs prepared using a mixture of $\mathrm{OxD}$ and $\mathrm{KeD}$ with different mass ratios of the two polymers was also studied. Similar to the encapsulation in OxDNCs, in the mixed system of OxDKeDNC, an increase in the apparent encapsulation efficiency of smaller payloads was observed in comparison to the encapsulation in $\mathrm{KeDNC}_{0.5}$ due to the presence of the polyaldehyde precursor. The results of the release from those OxDKeDNCs showed (Figure 6) that increasing the ratio of $\mathrm{OxD}$ in the mixture of functionalized dextran led to the synthesis of more stable NCs at a neutral $\mathrm{pH}$ value. However, those NCs were also less $\mathrm{pH}$-responsive than those prepared with more $\mathrm{KeD}$. The $\mathrm{NC} \mathrm{OxD}_{25} \mathrm{KeD}_{75} \mathrm{NC}_{0.5}$ displayed the best combination of long-term stability at a $\mathrm{pH}$ value of 7.4 and response to variations of the $\mathrm{pH}$ value of the environment.

The cytotoxicity of $\mathrm{ODNC}_{0.5}$ and $\mathrm{KDNC}_{0.5}$ was evaluated in HeLa cells using a luminescent cell viability assay (Figure 7A). Both the $\mathrm{OxDNC}_{0.5}$ and $\mathrm{KeDNC}_{0.5}$ did not display any cytotoxicity after 2 days of incubation even at concentrations as high as $300 \mu \mathrm{g} / \mathrm{mL}$. The cellular uptake of the NCs by HeLa cells was measured using NCs functionalized with cyanine-5 (Cy5), a fluorescent tag. Those NCs also encapsulated another fluorescent molecule, rhodamine-functionalized dextran $\left(M_{n}=\right.$ $500 \mathrm{kDa})$. The resulting fluorescent NCs were incubated for 2 , 24, or $48 \mathrm{~h}$ with HeLa cells, and after washing off the free NCs, the fluorescence of the cells was quantified by flow cytometry. 
Flow cytometry showed that both $\mathrm{OxDNC}_{0.5}$ and $\mathrm{KeDNC}_{0.5}$ were taken up by the cells (Figure $7 \mathrm{~B}$ ). The fluorescence intensity inside the cells increased with the coincubation time in keeping with the continuous uptake of the NCs during incubation. After $24 \mathrm{~h}$ of coincubation, ca. $60 \%$ of the HeLa cells had internalized $\mathrm{OxDNC}_{0.5}$ and ca. $45 \%$ of the cells contained $\mathrm{KeDNC}_{0.5}$ (Figure S10). Similar results were also observed with the mixed dextran NCs (Figure S11). Finally, confocal fluorescence microscopy was used to analyze the localization of the NCs in the cells to understand the fate of the $\mathrm{OxDNC}_{0.5}$ and $\mathrm{KeDNC}_{0.5}$ during their coincubation with the cells. The colocalization studies in the HeLa cells were performed with CellMask to identify the cellular membrane and LysoTracker to identify the lysosomes (Figure 6C). For most NCs taken up by the cells, both the rhodamine (from the cargo) and the CY-5 (from the NC shell) were present at the same location. Although the fluorescence signal from the NC shells coming from the NCs contained within the more acidic lysosomes seemed to overpower the signal from the cargo, to ascertain the release of the encapsulated payload from the NCs once in the lysosome, complementary release studies with payloads having a specific effect on the cellular function would be required.

\section{CONCLUSIONS}

In summary, we developed a robust and selective synthetic method for the preparation of $\mathrm{pH}$-responsive NCs. The bioorthogonal reaction between reactive carbonyl and hydrazide groups was used to generate the crosslinked hydrazone shell of the NCs, and the reaction occurred under biologically relevant conditions. The resulting NCs were successfully taken up by human HeLa cells without displaying any toxicity toward the cells. Additionally, encapsulated payloads were released by a variation in the $\mathrm{pH}$ value of the surrounding environment. The release was ascribed to the variation of the effective crosslinking density of the hydrazone network due to the dynamic equilibrium of the reaction between the reactive carbonyl and the hydrazide. The hydrazone crosslinking points dissociated in mildly acidic environments and this $\mathrm{pH}$ triggered change allowed for the controlled release of the payloads. The synthetic bio-orthogonal strategy used here can be expanded to other precursors and is an appealing tool to design controllable-release drug-delivery vehicles for the encapsulation of sensitive drugs. Because the new nanocarriers synthesized here were built using a bio-orthogonal reaction and were able to encapsulate macromolecular payloads successfully, they are ideal candidates for the delivery of sensitive biomacromolecular therapeutic agents such as protein, DNA, or RNA, and they could find applications in gene therapy, enzyme replacement therapy, vaccination, or cancer therapy.

\section{ASSOCIATED CONTENT}

\section{SI Supporting Information}

The Supporting Information is available free of charge at https://pubs.acs.org/doi/10.1021/acs.biomac.0c00492.

Detailed procedure for the synthesis of the nanocarriers precursors, additional details on the characterization of the nanocarriers, and complementary results obtained by DLS, electron microscopy, and flow cytometry (PDF)

\section{AUTHOR INFORMATION}

\section{Corresponding Authors}

Katharina Landfester - Max Planck Institute for Polymer Research, Mainz 55128, Germany; 이이이.org/0000-00019591-4638; Email: landfester@mpip-mainz.mpg.de

Héloïse Thérien-Aubin - Max Planck Institute for Polymer Research, Mainz 55128, Germany; 다이이.org/0000-00034567-516X; Email: therien@mpip-mainz.mpg.de

\section{Authors}

Mohammad Shafee Alkanawati - Max Planck Institute for Polymer Research, Mainz 55128, Germany

Richard da Costa Marques - Max Planck Institute for Polymer Research, Mainz 55128, Germany; Department of Dermatology, University Medical Center of the Johannes Gutenberg University Mainz, Mainz 55131, Germany

Volker Mailänder - Max Planck Institute for Polymer Research, Mainz 55128, Germany; Department of Dermatology, University Medical Center of the Johannes Gutenberg University Mainz, Mainz 55131, Germany; 이이.org/0000-00016583-8136

Complete contact information is available at:

https://pubs.acs.org/10.1021/acs.biomac.0c00492

\section{Notes}

The authors declare no competing financial interest.

\section{ACKNOWLEDGMENTS}

The authors thank Gunnar Glasser, Christoph Sieber, and Katrin Kirchhoff for their help with electron microscopy measurements. The financial support from the Max Planck Society and the Collaborative Research Centre 1066 of the DFG is acknowledged.

\section{REFERENCES}

(1) Ramil, C. P.; Lin, Q. Bioorthogonal chemistry: strategies and recent developments. Chem. Commun. 2013, 49, 11007-11022.

(2) Sletten, E. M.; Bertozzi, C. R. Bioorthogonal Chemistry: Fishing for Selectivity in a Sea of Functionality. Angew. Chem., Int. Ed. 2009, 48, 6974-6998.

(3) Lang, K.; Chin, J. W. Cellular Incorporation of Unnatural Amino Acids and Bioorthogonal Labeling of Proteins. Chem. Rev. 2014, 114, 4764-4806.

(4) Delaittre, G.; Goldmann, A. S.; Mueller, J. O.; Barner-Kowollik, C. Efficient Photochemical Approaches for Spatially Resolved Surface Functionalization. Angew. Chem., Int. Ed. 2015, 54, 11388-11403.

(5) Jiang, Y.; Chen, J.; Deng, C.; Suuronen, E. J.; Zhong, Z. Click hydrogels, microgels and nanogels: Emerging platforms for drug delivery and tissue engineering. Biomaterials. 2014, 35, 4969-4985.

(6) Algar, W. R.; Prasuhn, D. E.; Stewart, M. H.; Jennings, T. L.; Blanco-Canosa, J. B.; Dawson, P. E.; Medintz, I. L. The Controlled Display of Biomolecules on Nanoparticles: A Challenge Suited to Bioorthogonal Chemistry. Bioconjug. Chem. 2011, 22, 825-858.

(7) Piradashvili, K.; Paßlick, D.; Simon, J.; Höhner, J.; Mailänder, V.; Wurm, F.; Landfester, K. Fully Degradable Protein Nanocarriers by Orthogonal Photoclick Tetrazole-ene Chemistry for the Encapsulation and Release. Nanoscale. Horiz. 2017, 2, 297-302.

(8) Landfester, K.; Mailänder, V. Nanocapsules with specific targeting and release properties using miniemulsion polymerization. Expert. Opin. Drug. Deliv. 2013, 10, 593-609.

(9) Piradashvili, K.; Fichter, M.; Mohr, K.; Gehring, S.; Wurm, F. R.; Landfester, K. Biodegradable protein nanocontainers. Biomacromolecules. 2015, 16, 815-821.

(10) Mora-Huertas, C. E.; Fessi, H.; Elaissari, A. Polymer-based nanocapsules for drug delivery. Int. J. Pharm. 2010, 385, 113-142. 
(11) Utama, R. H.; Jiang, Y.; Zetterlund, P. B.; Stenzel, M. H. Biocompatible Glycopolymer Nanocapsules via Inverse Miniemulsion Periphery RAFT Polymerization for the Delivery of Gemcitabine. Biomacromolecules. 2015, 16, 2144-2156.

(12) Zhao, Y.; Lv, L.-P.; Jiang, S.; Landfester, K.; Crespy, D. Advanced stimuli-responsive polymer nanocapsules with enhanced capabilities for payloads delivery. Polym. Chem. 2015, 6, 4197-4205.

(13) Kuypers, S.; Pramanik, S. K.; D’Olieslaeger, L.; Reekmans, G.; Peters, M.; D'Haen, J.; Vanderzande, D.; Junkers, T.; Adriaensens, P.; Ethirajan, A. Interfacial thiol-isocyanate reactions for functional nanocarriers: a facile route towards tunable morphologies and hydrophilic payload encapsulation. Chem. Commun. 2015, 51, $15858-15861$.

(14) Fichter, M.; Piradashvili, K.; Pietrzak-Nguyen, A.; Pretsch, L.; Kuhn, G.; Strand, S.; Knuf, M.; Zepp, F.; Wurm, F. R.; Mailänder, V.; Landfester, K.; Gehring, S. Polymeric hepatitis $\mathrm{C}$ virus non-structural protein $5 \mathrm{~A}$ nanocapsules induce intrahepatic antigen-specific immune responses. Biomaterials. 2016, 108, 1-12.

(15) Crespy, D.; Stark, M.; Hoffmann-Richter, C.; Ziener, U.; Landfester, K. Polymeric Nanoreactors for Hydrophilic Reagents Synthesized by Interfacial Polycondensation on Miniemulsion Droplets. Macromolecules. 2007, 40, 3122-3135.

(16) Alkanawati, M. S.; Wurm, F. R.; Thérien-Aubin, H.; Landfester, K. Large-Scale Preparation of Polymer Nanocarriers by High-Pressure Microfluidization. Macromol. Mater. Eng. 2018, 303, 1700505.

(17) Piradashvili, K.; Alexandrino, E. M.; Wurm, F. R.; Landfester, K. Reactions and Polymerizations at the Liquid-Liquid Interface. Chem. Rev. 2016, 116, 2141-2169.

(18) Breitenkamp, K.; Emrick, T. Novel Polymer Capsules from Amphiphilic Graft Copolymers and Cross-Metathesis. J. Am. Chem. Soc. 2003, 125, 12070-12071.

(19) Roux, R.; Sallet, L.; Alcouffe, P.; Chambert, S.; SintesZydowicz, N.; Fleury, E.; Bernard, J. Facile and Rapid Access to Glyconanocapsules by CuAAC Interfacial Polyaddition in Miniemulsion Conditions. ACS. Macro. Lett. 2012, 1, 1074-1078.

(20) Malzahn, K.; Marsico, F.; Koynov, K.; Landfester, K.; Weiss, C. K.; Wurm, F. R. Selective Interfacial Olefin Cross Metathesis for the Preparation of Hollow Nanocapsules. ACS. Macro. Lett. 2014, 3, 4043.

(21) Cohen, J. D.; Zou, P.; Ting, A. Y. Site-Specific Protein Modification Using Lipoic Acid Ligase and Bis-Aryl Hydrazone Formation. Chem. Bio. Chem. 2012, 13, 888-894.

(22) Jiang, H.-P.; Liu, T.; Guo, N.; Yu, L.; Yuan, B.-F.; Feng, Y.-Q. Determination of formylated DNA and RNA by chemical labeling combined with mass spectrometry analysis. Anal. Chim. Acta. 2017, 981, 1-10.

(23) Nisal, R.; Jose, G. P.; Shanbhag, C.; Kalia, J. Rapid and reversible hydrazone bioconjugation in cells without the use of extraneous catalysts. Org. Biomol. Chem. 2018, 16, 4304-4310.

(24) Kalia, J.; Raines, R. T. Hydrolytic Stability of Hydrazones and Oximes. Angew. Chem., Int. Ed. 2008, 47, 7523-7526.

(25) Su, H.; Zhang, W.; Wu, Y.; Han, X.; Liu, G.; Jia, Q.; Shan, S. Schiff base-containing dextran nanogel as $\mathrm{pH}$-sensitive drug delivery system of doxorubicin: Synthesis and characterization. J. Biomater. Appl. 2018, 33, 170-181.

(26) Wang, S.; Nawale, G. N.; Oommen, O. P.; Hilborn, J.; Varghese, O. P. Influence of ions to modulate hydrazone and oxime reaction kinetics to obtain dynamically cross-linked hyaluronic acid hydrogels. Polym. Chem. 2019, 10, 4322-4327.

(27) Sonawane, S. J.; Kalhapure, R. S.; Jadhav, M.; Rambharose, S.; Mocktar, C.; Govender, T. AB2-type amphiphilic block copolymer containing a $\mathrm{pH}$-cleavable hydrazone linkage for targeted antibiotic delivery. Int. J. Pharm. 2020, 575, 118948.

(28) Gaudin, F.; Sintes-Zydowicz, N. Poly(urethane-urea) nanocapsules prepared by interfacial step polymerisation in miniemulsion: The droplet size: A key-factor for the molecular and thermal characteristics of the polymeric membrane of the nanocapsules? Colloids Surf. A. Physicochem. Eng. Asp. 2011, 384, 698-712.
(29) Kumar, A.; Kinneer, K.; Masterson, L.; Ezeadi, E.; Howard, P.; Wu, H.; Gao, C.; Dimasi, N. Synthesis of a heterotrifunctional linker for the site-specific preparation of antibody-drug conjugates with two distinct warheads. Bioorg. Med. Chem. Lett. 2018, 28, 3617-3621.

(30) Kölmel, D. K.; Kool, E. T. Oximes and Hydrazones in Bioconjugation: Mechanism and Catalysis. Chem. Rev. 2017, 117, 10358-10376.

(31) Larsen, D.; Kietrys, A. M.; Clark, S. A.; Park, H. S.; Ekebergh, A.; Kool, E. T. Exceptionally rapid oxime and hydrazone formation promoted by catalytic amine buffers with low toxicity. Chem. Sci. 2018, 9, 5252-5259.

(32) Larsen, D.; Pittelkow, M.; Karmakar, S.; Kool, E. T. New Organocatalyst Scaffolds with High Activity in Promoting Hydrazone and Oxime Formation at Neutral pH. Org. Lett 2015, 17, 274-277.

(33) Hafeez, S.; Ooi, H. W.; Morgan, F. L. C.; Mota, C.; Dettin, M.; Van Blitterswijk, C.; Moroni, L.; Baker, M. B. Viscoelastic Oxidized Alginates with Reversible Imine Type Crosslinks: Self-Healing, Injectable, and Bioprintable Hydrogels. Gels 2018, 4, 85.

(34) Kool, E. T.; Park, D.-H.; Crisalli, P. Fast Hydrazone Reactants: Electronic and Acid/Base Effects Strongly Influence Rate at Biological pH. J. Am. Chem. Soc. 2013, 135, 17663-17666.

(35) Masaro, L.; Zhu, X. X. Physical models of diffusion for polymer solutions, gels and solids. Prog. Polym. Sci. 1999, 24, 731-775. 